\title{
Video Head Impulse Test for Early Diagnosis of Vestibular Neuritis Among Acute Vertigo
}

\author{
Qiongfeng Guan, Lisan Zhang, Wenke Hong, Yi Yang, Zhaoying Chen, Peilin Lu, \\ Dan Zhang, Xingyue Hu
}

\begin{abstract}
Background: This study assesses the value of the video head impulse test (vHIT) for early diagnosis of vestibular neuritis (VN) among acute vertigo. Methods: Thirty-three cases of vestibular neuritis (VN), 96 patients with other acute vertigo (AV), and 50 cases of normal controls used vHIT to quantitatively test a pair of horizontal vestibulo-ocular reflection (VOR) gains, two pairs of vertical VOR gains, and the corresponding three pairs of VOR gain asymmetry. The peculiarity of VOR gains in VN and the differences between VN and other AV, normal controls by vHIT, were collected and analyzed. Results: There were statistically significant differences in the three pairs of VOR gains asymmetry between VN and other $\mathrm{AV}$, and normal controls $(P<0.01)$. The sensitivity was $87.9 \%$ and specificity was $94.3 \%$ in differentiating VN from normal and other acute vertigo by vHIT. Conclusions: This study shows vHIT has advantages in the diagnosis of $\mathrm{VN}$ in acute vertigo with good sensitivity and specificity and indicates a widespread clinical application.
\end{abstract}

RÉSUMÉ: Test vidéo d'impulsion de la tête dans le diagnostic précoce de la névrite vestibulaire chez les patients atteints de vertige aigu. Contexte: Au cours de cette étude nous avons évalué le test vidéo d'impulsion de la tête (vHIT) dans le diagnostic de la névrite vestibulaire (NV) chez les patients atteints de vertige aigu (VA). Méthodologie: Nous avons utilisé le vHIT pour évaluer quantitativement les gains d'une paire de reflexes vestibulooculaires (VOR) horizontaux, les gains de deux paires de VOR verticaux et l'asymétrie des gain des trois paires de VOR correspondants, chez 33 patients atteints de névrite vestibulaire, 96 patients présentant d'autres vertiges aigus et 50 sujets normaux. La particularité des gains de VOR dans la NV et les différences entre la NV et les autres VA et les sujets témoins au vHIT ont été notées et analysées. Résultats: Nous avons constaté qu'il existait des différences significatives au point de vue statistique dans l'asymétrie des trois paires de gains du VOR entre les patients atteints de NV et de VA, ainsi que des sujets témoins $(\mathrm{p}<0,01)$. La sensibilité était de $87,9 \%$ et la spécificité de $94,3 \%$ pour distinguer la NV de la normale et des autres vertiges aigus au moyen du vHIT. Conclusions: Cette étude démontre que le vHIT comporte des avantages dans le diagnostic de la NV chez les patients qui présentent un vertige aigu et que la sensibilité et la spécificité du test sont bonnes, ce qui appuie une utilisation généralisée du vHIT en clinique.

Keywords: acute vertigo, vestibular function, vestibular neuritis, vestibulo-ocular reflex, video head impulse test

doi:10.1017/cjn.2017.202

Can J Neurol Sci. 2017; 44: 556-561

Vertigo is the sensation of self-motion when no self-motion is occurring or the sensation of distorted self-motion during an otherwise normal head movement. The etiology of vertigo is hard to distinguish. The visual perception, proprioceptive, and vestibular system constitute the "equilibrial triad." The vestibular system is the primary mechanism in the maintenance of posture balance, and its pathological change is one of the main causes of vertigo. Currently, a sensitive and specific vestibular function test is not widely available in clinics. The video head impulse test (vHIT) is a quick and simple test for the examination of vestibular impairment, with a potentially clinical application. ${ }^{1-4}$ The test is mainly based on the principle of vestibulo-ocular reflex (VOR), and reflects vestibular function under high-frequency stimulation. ${ }^{5,6}$ Theoretically, the normal value of VOR is 1 . We used vHIT to investigate vestibular functions in patients with acute vertigo in an early stage to help with etiological diagnoses. We focused on the study of vestibular neuritis (VN), which is associated with a high morbidity rate and is easily confused with other vertigo. ${ }^{7} \mathrm{VN}$ primarily manifests as severe vertigo without hearing loss and is associated with peripheral vestibular impairment without involving the cochlea or central nervous system. It is still a diagnosis of exclusion. To distinguish VN from other vestibular syndrome was significant in the emergency department, and we used vHIT to determine if early diagnosis of $\mathrm{VN}$ might help patients to receive early treatment, such as glucocorticoids and antiviral agents. ${ }^{8}$

\section{METHODS \\ Patients}

Patients with dizziness and vertigo symptoms whose vestibular syndrome occurred within a week while without hearing loss were recruited from March 15, 2015, through September 10, 2015, and

From the Department of Neurology (QG, LZ, YY, PL, DZ, XH), Sir Run Run Shaw Hospital, School of Medicine, Zhejiang University, Hangzhou, China; Department of Neurology (QG, WH, ZC), Ningbo No. 2 Hospital, Ningbo, China.

Received May 19, 2016. Final Revisions Submitted March 26, 2017. Date of ACCEPTANCE APRIL 2, 2017.

Correspondence to: Xingyue Hu, Department of Neurology, Sir Run Run Shaw Hospital, School of Medicine, Zhejiang University, \#3 Qingchun East Road, Hangzhou, China. Email: huxingyue2003@126.com. 
were selected from the Sir Run Run Shaw Hospital affiliated with the Zhejiang University School of Medicine and Ningbo No. 2 hospital. Grouping was based on a final clinical diagnosis; exclusion would be done before hospital discharge in case of faulty diagnosis at admission. Finally, 33 patients with VN were selected, including 16 males and 17 females ages 28 to 70 years (mean, $53 \pm 14.9$ years). The total number of other acute vertigo (AV) patients was 96 , including 40 males and 56 females ages 26 to 82 years (mean, $53.0 \pm 15.1$ years). Forty-three patients were diagnosed benign paroxysmal positional vertigo, 17 males and 26 females, ages 26 to 78 years (mean, $48.2 \pm 11.5$ years). Psychogenic vertigo occurred in six females but no males. Pressure vertigo occurred in one female. Vertigo related to other systemic diseases was found in three males and three females. Paroxysmal vestibular disorders occurred in two females. Migraineassociated vertigo was observed in one female. Drug-induced vertigo was observed in one male. Epileptic vertigo occurred in one male. Posterior circulation infarction was observed in five males and three females. Uncertain vertigo concluded 12 males and 14 females. Normal controls were recruited from staff working at the Sir Run Run Shaw Hospital and Ningbo No.2 hospital, relatives of patients or volunteers, including 20 males and 30 females ages 21 to 81 years (mean, $50.5 \pm 12.4$ years). No history of tinnitus, deafness, dizziness, or balance impairment was recorded in the controls. No significant difference was observed in the male-to-female ratio or age among the $\mathrm{VN}$ and normal control group, AV group $(\mathrm{p}>0.05)$. Medical history and physical examination were assessed in all subjects. Severe vision damage with inability to focus on visual targets or impaired eye movement, intracranial surgery, or a history of ototoxic drugs was excluded. Hearing test of pure tone audiometry (PTA) was used in patients identified with possible decreased hearing. Considering the possibility of reduced hearing in the elderly, patients older than age 65 with PTA more than $40 \mathrm{~dB},{ }^{9}$ or less than 65 with PTA more than $25 \mathrm{~dB}$ were excluded. The present study was explained to patients and written informed consents were obtained. The protocol was approved by the Ethics Committee of the Sir Run Run Shaw hospital. The study conformed to ethical principles for medical research involving human subjects in accordance with the Declaration of Helsinki.

\section{Diagnosis of VN}

Clinical diagnostic criteria of $\mathrm{VN}^{10}$ were as follows: (1) acute or subacute onset of spontaneous vertigo occurred at least for more than 1 hour, or accompanied with nausea and vomiting; (2) spontaneous horizontal nystagmus, or accompanied with oscillating illusion of vision; (3) new-onset postural or imbalanced gait or accompanied with ataxia; (4) hearing impairment excluded by PTA; (5) no neurological deficit; (6) normal (e.g. changes related to age) or nonspecific abnormal magnetic resonance imaging-diffusion-weighted imaging (after symptoms for 1 to 10 days); (7) exclusion of previous history of vestibular dysfunction or impaired eye movement, acute drug/alcoholic poisoning, and recent head trauma; and (8) follow-up for at least more than 1 week. A final diagnosis was made by more than two neurologists with 10 years' clinical experience.

\section{vHIT Methods}

vHIT was used to examine all the patients and normal controls. An ICS Impulse ${ }^{\circledR}$ instrument of vHIT (GN Otometrics, Denmark) comprises computer analysis, a recording system, and an infrared video camera. The data acquisition system was obtained from a light texture eye mask placed on the patient's head and a video camera head placed on his or her right eye. During examination, the subject was in the sitting position at $1 \mathrm{~m}$ distance and was required to fix his or her gaze horizontally on a visual target. An experimenter stood behind the subject holding the subject's head. The subject was advised to relax the neck, and a head impulse test (HIT) was performed in each direction of the semicircular canal (SCC). Direction and timing of HIT were irregular and unpredictable. Peak head velocity of the impulses was gradually increased, ranging from $50^{\circ}$ to $250^{\circ}$ second (acceleration was $750-5000^{\circ} / \mathrm{s}^{2}$, amplitude was $5^{\circ}$ to $20^{\circ}$ ). At least 20 standard HITs were performed at each SCC and recorded by ICS impulse. HITs that did not meet the standard criteria were excluded from the tests. A pair of horizontal (left lateral/right lateral) VOR gains, and two pairs of vertical (left anterior/right posterior, and left posterior/right anterior) VOR gains were measured separately. Movement of a subject's head and eye during passive HIT was recorded and analyzed. One horizontal pair and two pairs of vertical VOR gains (gain = speed of eye movement/speed of head movement) and their bilateral asymmetry was automatically calculated separately. The final results for each subject were obtained from effective data of more than 15 HIT tests. We set the degree of dispersion ( $\sigma=$ standard deviation) at less than 0.10 in the horizontal plane and less than 0.15 in the vertical plane to ensure accuracy and consistency of the data.

\section{Statistical Analysis}

Statistical analysis was performed using SPSS 20 (IBM Corp., Armonk, NY). Measurement of VOR gain and asymmetry data was summarized as mean \pm standard deviation and verified by normal distribution. Count data such as number of positive or negative results were compared with a two-tailed $t$ test $($ alpha $=0.01)$.

\section{RESUltS}

\section{Results of VOR Gains and Asymmetry in the Normal Control (NC) Group}

The VOR gains were infinitely close to 1 , particularly in the horizontal semicircular canal. No significant difference was observed in VOR gains and asymmetry among different SCC ( $>$ 0.05; Table 1, NC group).

\section{Results of VOR Gains and Asymmetry in Different Groups}

Significant difference was found in every VOR gain asymmetry between the VN group and NC group (*p $<0.001$, Table 1$)$. Significant difference was found in every VOR gain asymmetry between the VN group and AV group (***p $<0.001$, Table 1). A statistical difference was found in every VOR gain asymmetry between the AV and the NC groups (***p $<0.001$, Table 1$)$, with close average values, but it was not enough to distinguish abnormal results from artificial error. Except for one case that could not be distinguished, 22 cases involving horizontal SCC function loss were defined as superior vestibular nerve dysfunction, seven cases involving posterior SCC function loss were defined as inferior vestibular nerve dysfunction and three cases involving both were analyzed separately. Significant differences in the affected side of lateral VOR gains were observed between the superior vestibular 
Table 1: Comparison of vHIT VOR gains and asymmetry results within VN group, NC group, and AV group

\begin{tabular}{|c|c|c|c|c|c|c|c|c|c|}
\hline Group & LL & RL & Asymmetry \% & LA & RP & Asymmetry \% & LP & RA & Asymmetry \% \\
\hline $\mathrm{NC}^{\dagger}$ & $0.99 \pm 0.08$ & $0.98 \pm 0.16$ & $4.18 \pm 3.03$ & $0.99 \pm 0.11$ & $0.99 \pm 0.10$ & $5.08 \pm 4.15$ & $0.89 \pm 0.09$ & $0.94 \pm 0.11$ & $7.42 \pm 5.20$ \\
\hline $\mathrm{VN}^{\ddagger}$ & $0.72 \pm 0.25^{* * * *}$ & $1.02 \pm 0.17$ & $30.31 \pm 18.80^{* * * *}$ & $0.83 \pm 0.25^{*, * *}$ & $0.84 \pm 0.15^{* * * *}$ & $14.84 \pm 12.56^{* * * *}$ & $0.78 \pm 0.23^{* * * *}$ & $0.91 \pm 0.23$ & $19.28 \pm 16.86^{*, * *}$ \\
\hline $\mathrm{sVN}^{\ddagger}$ & $0.60 \pm 0.17^{* * * *}$ & $1.01 \pm 0.17$ & $40.50 \pm 12.42 * * *$ & & & & & & \\
\hline $\mathrm{iVN}^{\frac{1}{3}}$ & & & & & & & $0.57 \pm 0.12^{* * * *}$ & $0.93 \pm 0.19$ & $36.50 \pm 12.10^{* * * *}$ \\
\hline $\mathrm{AV}^{\ddagger}$ & $1.01 \pm 0.13$ & $1.02 \pm 0.15$ & $5.66 \pm 5.26 * * *$ & $1.08 \pm 0.64$ & $1.01 \pm 0.17$ & $8.16 \pm 5.86^{* * *}$ & $0.89 \pm 0.45$ & $0.94 \pm 0.18$ & $12.02 \pm 6.91 * * *$ \\
\hline
\end{tabular}

Values are mean gain \pm standard deviation $(n=50$ in the NC group, $n=33$ in the VN group, $n=96$ in the AV group). The data were analyzed by a two-tailed $t$ test. *p $<0.001 \mathrm{VN}$ vs NC groups. **p $<0.001 \mathrm{VN}$ vs AV groups. ***p $<0.05 \mathrm{VN}$ vs NC groups. The pairs were matched based on sequences if the patients were positioned according to disease condition.

${ }^{\dagger}$ The VOR gains and asymmetry in the NC group were calculated in LL/RL, LA/RP, and LP/RA pairs.

${ }^{*}$ The VOR gains and asymmetry in the VN group were calculated based on affected lateral/intact lateral, affected anterior/intact posterior, and affected posterior/intact anterior.

Benign paroxysmal positional vertigo patients were the same as the VN group based on niveau (left or right side). Asymmetry was location independent. Except for one case that could not be distinguished, 22 cases involving horizontal SCC function loss were defined as sVN dysfunction, seven cases involving vertical SCC function loss were defined iVN, and three cases involving both were analyzed separately.

iVN, inferior vestibular nerve; LA, left anterior; LL, left lateral; LP, left posterior; RA, right anterior; RL, right lateral; RP, right posterior; sVN, superior vestibular nerve.

nerve and AV group, and NC group $(\mathrm{p}<0.01)$. Significant differences in the affected side of posterior VOR gains were observed between the inferior vestibular nerve and AV group, and NC group $(p<0.01)$. Despite significant differences in the affected side of VOR, gains both in horizontal and vertical were observed in the whole VN group, the absolute value of vertical VOR gain is not as accurate and stable as VOR gain asymmetry for the artificial error reason.

\section{Sensitivity and Specificity of vHIT in the Diagnosis of VN}

According to the previous literature and the results of our repeated tests, $20 \%$ or more in horizontal or $25 \%$ or more in vertical

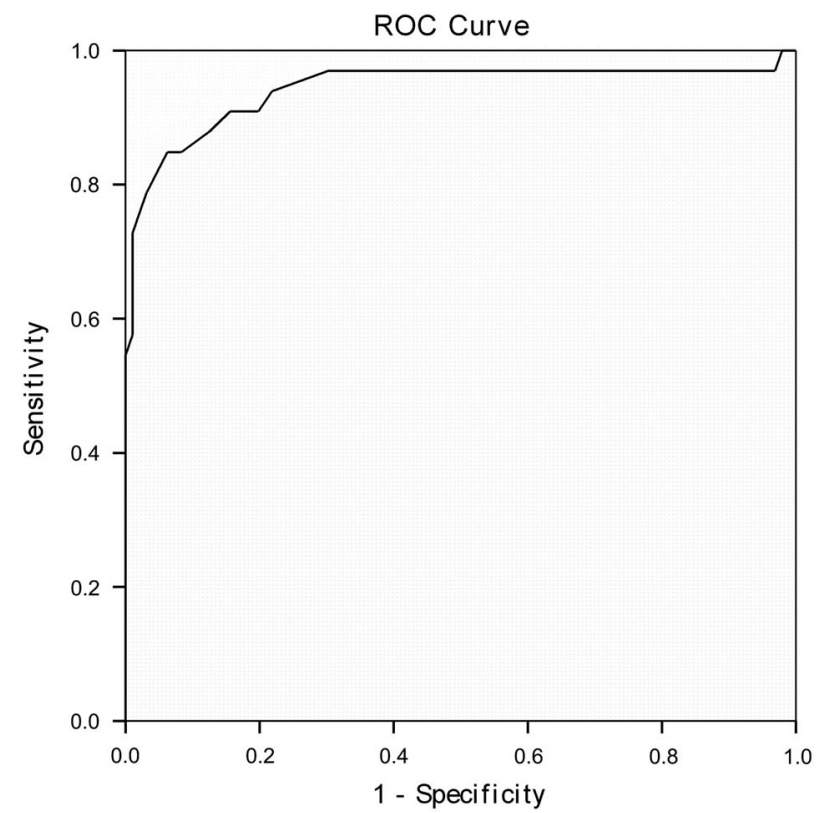

Figure 1: The receiver operating characteristic curve of sensitivity and specificity of VOR gain asymmetry. Area, $0.943 \pm 0.031$ (95\% confidence interval, 0.882-1.000).
VOR gain asymmetry were defined as positive and less than $20 \%$ horizontal or less than $25 \%$ vertical VOR gain asymmetry as negative. The VOR gain asymmetry in VN group were calculated based on affected lateral/intact lateral, affected anterior/intact posterior and affected posterior/intact anterior. Positive results were found in 21 cases with horizontal VOR gain asymmetry, five cases with vertical VOR gain asymmetry, and three in both. Negative results were found in four cases of VN. The sensitivity of vHIT was $87.9 \%$ and the specificity was $94.8 \%$ in differentiating VN from normals and other acute vertigo; positive predictive value was $85.3 \%$ and negative predictive value was $95.8 \%$. The receiver operating characteristic curve is shown in Figure 1.

\section{DISCUSSION}

$\mathrm{VN}$ is an acute unilateral peripheral vestibular disease without other dysfunctions or signs of brain stem involvement. Currently, clinical diagnosis of $\mathrm{VN}$ is based on exclusion. Based on our results, we conclude that vHIT is a useful tool that can be used to detect $\mathrm{VN}$ patients in the acute phase in an emergency department. We found the VOR gain asymmetry values were significantly different from other AV patients and normal controls, with a high sensitivity (87.9\%) and specificity (94.8\%), the area under the receiver operating characteristic curve was 0.943 , this indicated that vHIT might be a reliable method for diagnosis of VN. Compared with bedside HIT, vHIT requires a relatively smaller head impulse movement and it can be widely used for clinical assessment in the elderly and children, particularly, for bedside examination. $^{1-4,11,12}$ Supportive evidence using a larger sample size may alter the approach to early clinical diagnosis of $\mathrm{VN}$ in future.

The vHIT examination to test VOR in three pairs of SSC was not long. Because it was easy and quick for operation, the researchers and doctors expected it could be widely used in the clinic. Comparing vHIT and caloric testing in the diagnosis of peripheral vestibular disease, Bartolomeo et al. ${ }^{13}$ found that the sensitivity was $68.84 \%$ and the specificity was $100 \%$ at the caloric testing value of $30 \%$. McCaslin et al. ${ }^{14}$ showed the sensitivity was $78 \%$ and specificity was $95 \%$. These results showed that vHIT 
specificity was very high but sensitivity was relatively low. In the present study, we demonstrated that vHIT in acute vertigo was ideal for a sensitive and specific diagnosis of $\mathrm{VN}$, with a high sensitivity of $87.9 \%$. Mahringer et al. ${ }^{15}$ considered that sensitivity at acute phase was $61 \%$ and only $33 \%$ at nonacute phase. The VN patients in our test were all in acute phase and the average time from onset to testing was only 3.6 days, which increased the positive results and might enhance the sensitivity of vHIT. Another reason was that all subjects were screened for hearing. Some of the peripheral vestibular disorders with hearing impairment were included in the previous study, for example, Ménière's disease might have normal, increased, or decreased VOR, ${ }^{16-18}$ which would lead to a significant increase in false-positive results. A minority of patients with VN may have high-frequency hearing damage $^{19}$ with only a slight decrease of 16 to $24 \mathrm{~dB}$. Currently, $\mathrm{VN}$ is not considered involved in damage to the cochlear nerve. We believe PTA screening is necessary for patients with suspicious hearing impairment, which will significantly increase the objective and specificity of diagnosis. Three negative results of our tests actually approached the cutoff of asymmetry for the diagnosis. In one case, the horizontal asymmetry was $18 \%$. Vertical asymmetry in the remaining two cases was $19 \%$ and $21 \%$, respectively; this indicated that the results were close to the cutoff needed to be carefully treated based on clinical features. In another case, the VOR gain asymmetry from three matched pairs was $2 \%$ and symptoms of the patient disappeared quickly, although we did the test on the second day. So far, the specificity of vHIT in differential diagnosis of vertebrobasilar stroke in acute vestibular syndrome was highly evaluated, its accuracy rate of vHIT could reach $91 \%$ to $100 \% .^{3,20,21}$ Our results contained five patients with vertebrobasilar stroke and their VOR results were all negative; however, they must be judged based on clinical features rather than relying on the vHIT results merely. The VOR gain asymmetry between $20 \%$ and $30 \%$ is still controversial until now. ${ }^{3}$
A

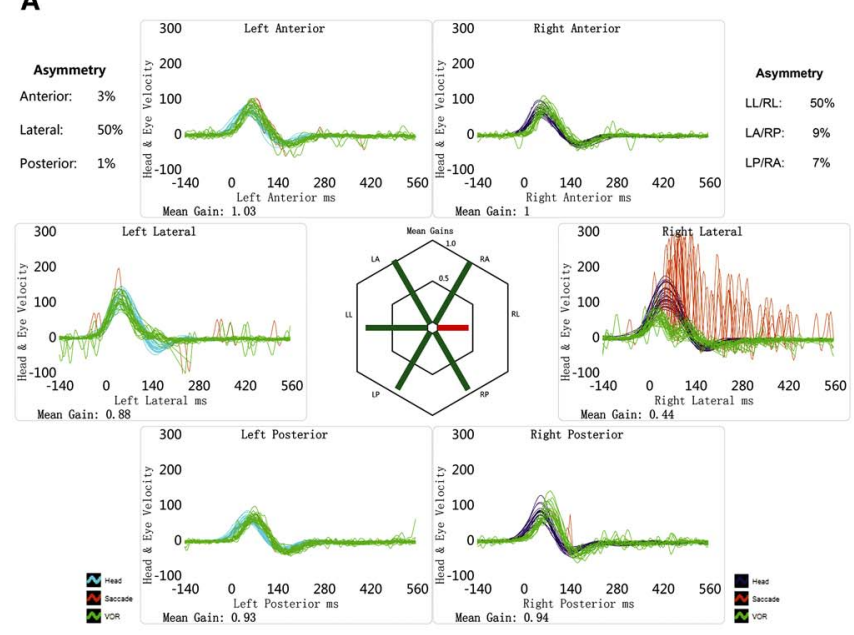

C

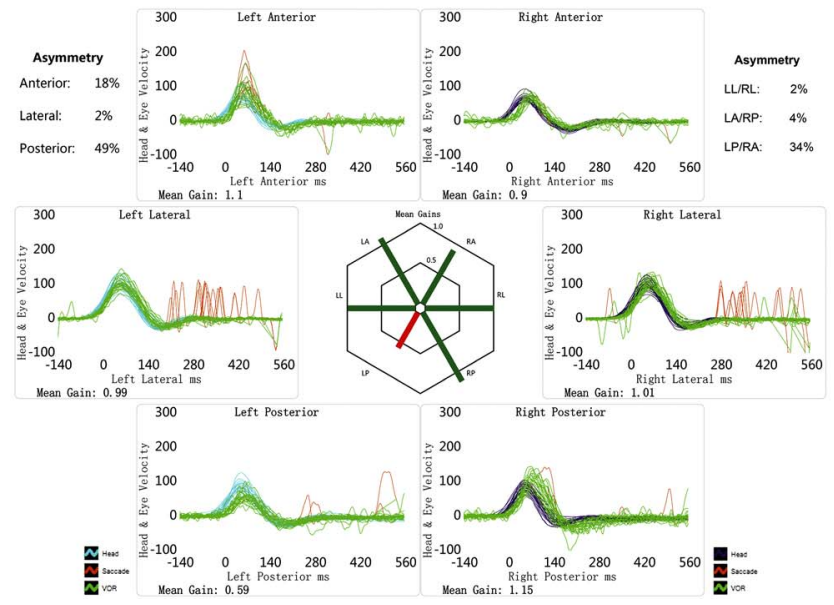

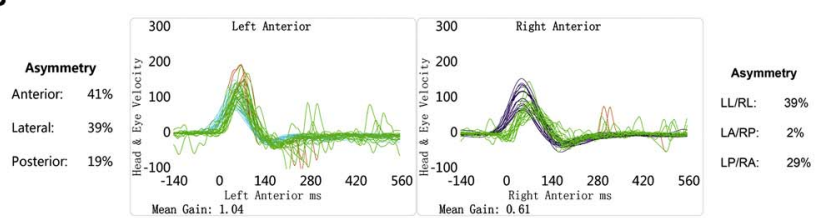

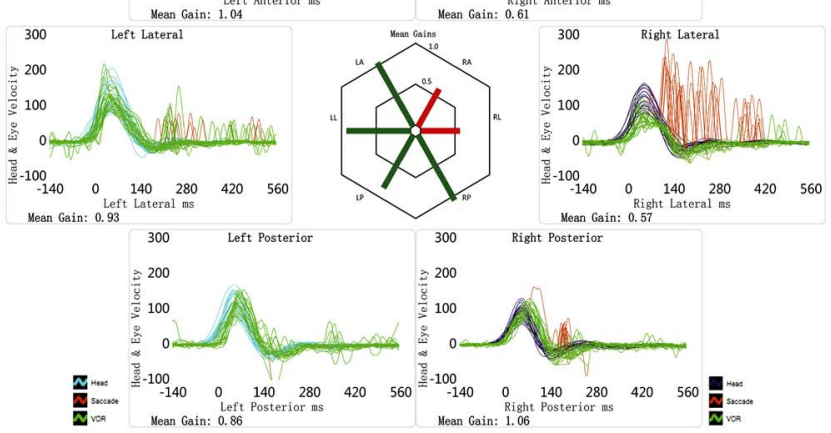

D
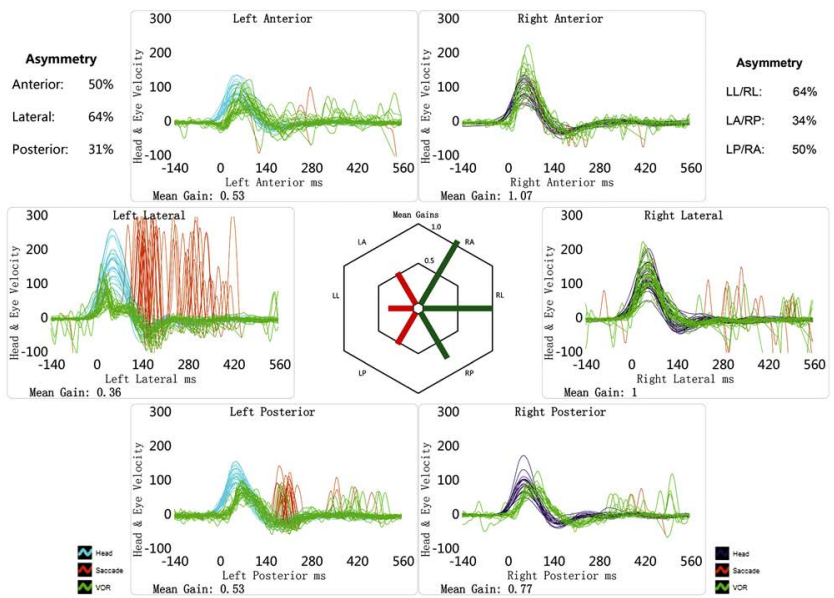

Figure 2: vHIT results in different types of VN patients. (A. Superior VN, patient 1. this showed a significantly reduced VOR gain in the right lateral SCC compared with left lateral SCC. The asymmetry was 50\% between the affected and the intact side. (B) Superior VN, patient 2. It showed a significantly reduced VOR gain in the right lateral and anterior SCC compared with left lateral and posterior SCC; the asymmetries were 39\% and $29 \%$, respectively. (C) Inferior VN, patient 3. It showed a significantly reduced VOR gain in left posterior SCC compared with right anterior SCC, with an asymmetry of 34\%. (D) VN patient 4 with both the superior and inferior vestibular nerve involvement. This showed a significantly reduced VOR gain in all left SCCs compared with the right side; the asymmetry was, respectively, 64\%, 34\%, and 50\%. In a Hex plot of mean gain (in the middle of the image), red=mean gain $<$ unilateral cutoff $(0.8$ in lateral SCC or $<0.7$ in anterior or posterior SCC), green = mean gain between unilateral cutoff and 1.2. In the VOR analysis, red $=$ saccade, green $=$ VOR, blue or purple $=$ head movement . 
In contrast to any previous vestibular function tests, vHIT enables the location of vestibular damage and detection of vertical SCC function. ${ }^{22,23}$ It has traditionally been thought that VN involves the superior vestibular nerve only. ${ }^{24}$ Results of the scleral search coil technique found that $\mathrm{VN}$ can also involve the inferior vestibular nerve alone; ${ }^{25}$ therefore, acute peripheral vestibular damage can be divided into three types, which can also be tested by vHIT: (1) horizontal SCC functional loss or horizontal and anterior SCC functional loss; (2) horizontal, anterior, and posterior SCC functional loss; and (3) simple posterior SCC function loss. Halmagyi et al. ${ }^{26}$ reported selective inferior VN patients, whereas horizontal SCC functional loss was not essential for diagnosis of VN. Inferior VN can detect posterior SCC functional loss, which is detected as normal function by caloric testing. ${ }^{27,28}$ We summarized our positive results: 15 suffered horizontal SCC functional loss only (Figure 2A), six suffered both horizontal and anterior SCC functional loss (Figure 2B), five involved posterior SCC functional loss (Figure 2C), and three involved the whole SCC (Figure 2D). We believe that increased application of vHIT and data acquisition will facilitate further investigation of the mechanisms of VN. The absolute value of vertical VOR gain is affected greatly by systematic errors, such as patient compliance according to our repeated tests; it might move up or down in pairs in vertical SCC, whereas the VOR gain asymmetry was relatively stable and accurate. To reduce artificial errors, the velocities were mostly controlled below $50^{\circ}$ to $200 \%$ second in our research. The lower velocities, especially on vertical SCC, could lose a slight true positive; therefore, suspicious results around cutoff might need to be retested. We look forward more researchers undertaking more tests to explore this issue.

Another advantage of vHIT is that it could record catch-up saccades sensitively, which indicates damages associated with high-frequency vestibular function. In contrast to the low levels of physiological saccade amplitude, the abnormal catch-up saccades not only showed a high amplitude peak speed, but also an increase in speeding head impulse; the positive rate of abnormal catch-up saccades markedly increases in patients with vestibular function impairment. ${ }^{29,30}$ All VN patients in our study were observed with high amplitude catch-up saccades; $24 \%$ patients in the AV group also recorded continuous catch-up saccades, with mostly a lower peak. Another interesting point of the results was that saccades were usually recorded in lateral image rather than vertical image, even if the vertical canals were affected heavily. Although some researchers have tried to quantitate saccades, ${ }^{31}$ it has been difficult to determine the standard to distinguish the results. The positive rate also increased with degeneration factor in the elderly; ${ }^{11}$ therefore, the role of catch-up saccades in vestibular dysfunction still needs to be investigated in further studies.

In conclusion, this study has shown that vHIT is a simple, quick, and safe test that can mensurate VOR gains in all six semicircular canals quantitatively. The VOR gain asymmetry was relatively more accurate than absolute value of VOR gain, especially in the vertical. It displays good sensitivity and specificity in the early diagnosis of VN associated with acute vertigo without hearing impairment. The results around the cutoff of asymmetry should be treated cautiously.

\section{ACKNOWLEDGMenTs AND Funding}

The study was supported by the Zhejiang Medical Science and Technology Project Foundation (2014KYB136 and 2016KYB264) and the Zhejiang Province Natural Science Foundation (LY15H090004).

\section{Disclosures}

QG reports grants from Zhejiang Medical Science and Technology Project Foundation (2016KYB264) outside the submitted work; LZ reports grants from Zhejiang Medical Science and Technology Project Foundation (2014KYB136) and from Zhejiang Province Natural Science Foundation (LY15H090004) outside the submitted work. The remaining authors have nothing to disclose.

\section{Statement of Authorship}

XH contributed to design of the study; QG performed the study and executed all the video head impulse tests; $\mathrm{ZC}$ analyzed the data; LZ, WH, PL, and DZ evaluated and treated the patients; $\mathrm{LZ}$ and YY performed the analysis with constructive discussions.

\section{REFERENCES}

1. Weber KP, MacDougall HG, Halmagyi GM, et al. Impulsive testing of semicircular-canal function using video-oculography. Ann N Y Acad Sci. 2009;1164:486-91.

2. MacDougall HG, Weber KP, McGarvie LA, et al. The video head impulse test: diagnostic accuracy in peripheral vestibulopathy. Neurology. 2009;73:1134-41.

3. Newman-Toker DE, Saber Tehrani AS, Mantokoudis G, et al. Quantitative video-oculography to help diagnose stroke in acute vertigo and dizziness: toward an ECG for the eyes. Stroke. 2013;44:1158-61.

4. Armato E, Ferri E, Pinzani A, Ulmer E. Cerebellar haemorrhage mimicking acute peripheral vestibulopathy: the role of the video head impulse test in differential diagnosis. Acta Otorhinolaryngol Ital. 2014;34:288-91

5. Halmagyi GM, Curthoys IS. A clinical sign of canal paresis. Arch Neurol. 1988;45:737-9.

6. Grossman GE, Leigh RJ, Bruce EN, et al. Performance of the human vestibuloocular reflex during locomotion. J Neurophysiol. 1989;62:264-72.

7. Strupp M, Brandt T. Vestibular neuritis. Semin Neurol. 2009;29:509-19.

8. Strupp M, Zingler VC, Arbusow V, et al. Methylprednisolone, valacyclovir, or the combination for vestibular neuritis. N Engl J Med. 2004;351:354-61.

9. Ventry IM, Weinstein BE. The hearing handicap inventory for the elderly: a new tool. Ear Hear. 1982;3:128-34.

10. Mantokoudis G, Tehrani AS, Wozniak A, et al. VOR gain by head impulse video-oculography differentiates acute vestibular neuritis from stroke. Otol Neurotol. 2015;36:457-65.

11. Agrawal Y, Schubert MC, Migliaccio AA, et al. Evaluation of quantitative head impulse testing using search coils versus video-oculography in older individuals. Otol Neurotol. 2014;35: 283-8.

12. Wolter NE, Gordon KA, Papsin BC, et al. Vestibular and balance impairment contributes to cochlear implant failure in children. Otol Neurotol. 2015;36:1029-34.

13. Bartolomeo M, Biboulet R, Pierre G, et al. Value of the video head impulse test in assessing vestibular deficits following vestibularneuritis. Eur Arch Otorhinolaryngol. 2014;271:681-8.

14. McCaslin DL, Jacobson GP, Bennett ML, et al. Predictive properties of the video head impulse test: measures of caloric symmetry and self-report dizziness handicap. Ear Hear. 2014;35:e185-91.

15. Mahringer A, Rambold HA. Caloric test and video-head-impulse: a study of vertigo/dizziness patients in a community hospital. Eur Arch Otorhinolaryngol. 2014;271:463-72.

16. Manzari L, Burgess AM, MacDougall HG, et al. Rapid fluctuations in dynamic semicircular canal function in early Meniere's disease. Eur Arch Otorhinolaryngol. 2011;268:637-9.

17. Maire R, van Melle G. Vestibulo-ocular reflex characteristics in patients with unilateral Meniere's disease. Otol Neurotol. 2008;29:693-8. 
18. Zulueta-Santos C, Lujan B, Manrique-Huarte R, et al. The vestibuloocular reflex assessment in patients with Meniere's disease: examining all semicircular canals. Acta Otolaryngol. 2014;134:1128-33.

19. Rahko T, Karma P. New clinical finding in vestibular neuritis: highfrequency audiometry hearing loss in the affected ear. Laryngoscope. 1986;96:198-9.

20. Newman-Toker DE, Kattah JC, Alvernia JE, et al. Normal head impulse test differentiates acute cerebellar strokes from vestibular neuritis. Neurology. 2008;70:2378-85.

21. Mantokoudis G1, Tehrani AS, Wozniak A, et al. VOR gain by head impulse video-oculography differentiates acute vestibular neuritis from stroke. Otol Neurotol. 2015;36:457-65.

22. Halmagyi GM, Aw ST, Cremer PD, et al. Impulsive testing of individual semicircular canal function. Ann N Y Acad Sci. 2001;942:192-200

23. MacDougall HG, McGarvie LA, Halmagyi GM, et al. Application of the video head impulse test to detect vertical semicircular canal dysfunction. Otol Neurotol. 2013;34:974-9.

24. Fetter M, Dichgans J. Vestibular neuritis spares the inferior division of the vestibular nerve. Brain. 1996;119:755-63.
25. Aw ST, Fetter M, Cremer PD, et al. Individual semicircular canal function in superior and inferior vestibular neuritis. Neurology. 2001;57:768-74.

26. Halmagyi GM, Aw ST, Karlberg M, et al. Inferior vestibular neuritis. Ann N Y Acad Sci. 2002;956:306-13.

27. Monstad P, Okstad S, Mygland A. Inferior vestibular neuritis: 3 cases with clinical features of acute vestibular neuritis, normal calorics but indications of saccular failure. BMC Neurol. 2006;6:45.

28. Taylor RL, McGarvie LA, Reid N. Vestibular neuritis affects both superior and inferior vestibular nerves. Neurology. 2016;87:1704-12.

29. Weber KP, Aw ST, Todd MJ, McGarvie LA, Curthoys IS, Halmagyi GM. Head impulse test in unilateral vestibular loss: vestibuloocular reflex and catch-up saccades. Neurology. 2008;70:454-63.

30. Blodow A, Pannasch S, Walther LE. Detection of isolated covert saccades with the video head impulse test in peripheral vestibular disorders. Auris Nasus Larynx. 2013;40:348-51.

31. Chen L, Todd M, Halmagyi GM, et al. Head impulse gain and saccade analysis in pontine-cerebellar stroke and vestibular neuritis. Neurology. 2014;83:1513-22. 